\title{
Editorial
}

\section{Congenital heart disease in adults and adolescents}

\author{
JANE SOMERVILLE \\ From the Paediatric and Adolescent Unit, National Heart Hospital, London
}

Without treatment only about $10-15 \%$ of children born with congenital anomalies of the heart or vascular system would survive beyond puberty. Times have changed since the days when congenita morbus cordis and morbus caerulia were the definitive diagnoses on case notes of patients with untreated cyanotic and acyanotic congenital heart disease; without treatment $20-25 \%$ died in the neonatal period and $50-60 \%$ died in the first year. The impact of surgical treatment for congenital heart disease stimulated the growth of specialist paediatric cardiology and with refined diagnostic ability, improved anaesthesia, and intensive care came a clearer understanding of cardiac anatomy that resulted in a revolution in the once threatened and limited lives of such patients.

Perhaps unexpectedly, all this endeavour and progress has increased rather than decreased the number of adolescents and adults who need advice on problems associated with their congenital cardiac anomalies. This patient population is made up of "natural" survivors with mild or more serious compensated lesions who have "escaped" surgical treatment, not needed it, or not been suitable for it and a larger group who are the long term survivors of earlier surgical palliation or radical repair of their lesions, euphemistically referred to as "total correction".

The most frequently encountered natural survivors are those with the mild valve lesionsbicuspid or fused tricuspid aortic valves that calcify or are a site for endocarditis; mitral valve anomalies such as ballooned floppy valves; and often familial or, less frequently, mild or moderate pulmonary valve stenosis. Small or moderate sized ventricular septal defects may be discovered when endocarditis develops, and in such cases the diagnosis is usually

Requests for reprints to Dr Jane Somerville, Paediatric and Adolescent Unit, National Heart Hospital, Westmoreland Street, London W1M 8BA. overdue; less frequently atrial fibrillation causes symptoms in those with a left ventricular-right atrial shunt. The discovery of atrial septal defects, secundum and less commonly primum (atrioventricular defects), may be prompted by the development of arrhythmias, and sometimes a small duct presents as atrial fibrillation or as an unusual chest radiograph. Routine examinations for insurance, and employment purposes, or during pregnancy are the usual disclosers of unsuspected congenital lesions of the heart. Even coarctation of the aorta occasionally escapes diagnosis in childhood. More complex lesions such as Ebstein's anomaly, corrected transposition, and common atrium that have been recognised during childhood but not treated, may later cause symptoms associated with established arrhythmias, endocarditis, or paradoxical emboli after abdominal or other operation.

Cyanotic congenital heart disease in adults requires special care and understanding. Because there are additional problems management is not as simple as suggested in textbooks of paediatric cardiology. The compensatory polycythaemia that develops in adult cyanotic patients may cause symptoms that can be relieved by controlled venesection and appropriate volume replacement. But too often treatment is overzealous or volume replacement is neglected. In such patients thrombosis or haemorrhage readily develop in response to trauma. They may have gout (which may still be diagnosed as whitlows in some casualty departments); renal problems leading to anuria in response to fluid restriction; skin sepsis from chronic acne; and chronic arthropathy in the ankles and knees, which may be their major complaint. Relative anaemia, which may be caused by excessive venesection, gastric bleeding, or bad nutrition, may escape notice and cause worsening symptoms or heart failure in sinus rhythm. Routine cardiac catheterisation and angiography carry special risks, and unless they are scrupulously planned and performed may not pro- 
vide the desired information. Such investigations should not be performed by an inexperienced but enthusiastic investigator because complications are frequent.

After the patient reaches the age of 25 years the stenosed pulmonary valve calcifies, and if the ventricular septum is intact there may be associated calcification in the tricuspid annulus. In adults with Fallot's tetralogy the calcified pulmonary valve may need to be replaced, aortic regurgitation is a common and under recognised problem, and the frequent occurrence of prolonged arterial desaturation after radical repair causes anaesthetists to delay extubation unnecessarily. Chronic right heart failure and infection, haemorrhage, and renal complications are more common than they are in children with the same cyanotic diseases.

The patients who are most dependent on expert cardiological care for continued life are those with the Eisenmenger reaction. The length and quality of their lives are profoundly influenced by advice and decisions surrounding events that are routine in normal individuals. General anaesthesia, pregnancy, use of oral contraceptives, minor surgery, trauma with haemorrhage, and living at a high altitude are hazardous. Junior anaesthetic and surgical staff should not manage appendicectomy, tooth extraction, and other minor procedures in patients with the Eisenmenger reaction. Cardiologists should be called in early to coordinate the management of such patients with other senior staff. Pregnancy is contraindicated but it is not necessarily appropriate to advise sterilisation of the male partner. Each woman requires an individual solution which, despite the risks of operation, may be sterilisation. Fortunately there are fewer of these patients than there were 20 years ago, probably because many serious lesions are now surgically treated in infancy before the Eisenmenger reaction develops. Heart and lung transplantation offers new hope for the select few; and it is important that there are no mistakes in early management, such as unnecessary thoracotomies, over zealous treatment of polycythaemia with radioactive phosphorus, or delayed recognition of cerebral abscess.

There is special provision for adolescents and young adults with congenital heart disease in the National Heart Hospital. An adolescent unit caters for the increasing numbers of young patients who have had surgical treatment and will require further operation or specialised medical treatment. Here young people are nursed away from older patients with degenerative and terminal disease and can enjoy noise, talk, and different television programmes and yet be separate from infants and toddlers.
Patients with arrhythmias (which are often complex), pacemakers, chronic myocardial dysfunction, effects of progressive pulmonary vascular disease after closure of defects, and endocarditis require inpatient treatment. Postoperative infective endocarditis, other than in patients with valve replacements or aortic and mitral valve disease, is relatively uncommon. When it does strike, diagnosis is too often dangerously delayed or distorted. Here blame must be laid on family doctors who treat fever in young cardiac patients with antibiotics, and ignore repeated advice and hospital cards that warn against the use of antibiotics before blood has been taken for culture. It is difficult to get the message understood by the complacent. It is equally important to draw attention to the lesions that do not require antibiotic protection from endocarditis such as pulmonary valve stenosis, closed atrial and ventricular septal defects without left sided lesions, and surgically closed ducts.

The largest demand for inpatient care comes from those requiring reoperation because of inadequate surgery, progress of trivial lesions, damage by endocarditis, degeneration or dysfunction of valve replacements, progressive calcification of the abnormal aortic valve, and radical repair (or palliation) after shunts or bands that no longer relieve symptoms. This often comes as a daunting surprise to patients and relatives who have not been prepared for the possibility of further operation. Resentment is frequent, and this sometimes increases the difficulties of appropriate management.

Risks of reoperation are usually higher than with the first operation. Means to prevent valve calcification and degeneration of bioprostheses or homografts are urgently needed. The track record of the properly chosen homograft in terms of durability and maintained function, though not perfect, is much better than that of any other bioprosthesis. The most difficult problems come from patients who have complex congenital heart disease such as tricuspid atresia, some pulmonary atresias, and double inlet ventricles (single, uni, and common) kept alive by earlier and often multiple palliative operations. Sometimes the previous operation has wrecked the pulmonary arteries or a deterioration in myocardial function precludes further successful radical palliation. These longterm disasters may well have been initially reported as surgical triumphs, and with earlier informed guidance some of these disasters might have been averted. Cardiac or cardiopulmonary transplantation may now offer the only solution. Alas, the earlier attempts at palliation, particularly those that do not produce effective arteriopulmonary anastomoses, may prevent the success of even this "last dash". 
Many young patients with treated or compensated congenital heart disease are not disabled, but they may require expert advice about employment, careers, insurance, driving licences, pregnancy, contraception, and genetic risk to offspring. Those who break the law may claim that their heart disease is a mitigating factor and before imposing a prison sentence a judge should seek medical guidance on the severity and prognosis of the disease. Medicolegal guidelines regarding driving have been updated and to some extent liberalised. Further clarification on the issue of suitability of young adults with treated and untreated congenital heart disease to hold licences for heavy goods vehicles or public service vehicles is required. New recommendations are awaited.

Medical progress has produced a new medical community. Alas, the long term needs and care of these people have not been adequately considered. Much time and money have already been invested to achieve their survival. They are young and most are anxious to play a normal role in society. Some of those who have had heart operations are unfairly penalised when seeking employment. Their lot can be enhanced by expert medical guidance and supervision.

Who should take care of this new medical community? The paediatric cardiologist? The cardiologist? A new subspecialist? It is difficult to stimulate the interest of those responsible for cardiological services and education because this group of patients is small and has limited requirements compared with the larger demands of those with coronary disease or hypertension. The training and technical expertise of the paediatric cardiologist are not designed for the management of adult patients and their special needs. But he understands the disease as it starts, presents, and is treated, and some of its complications. Managing these patients until they are 20 years old, as some paediatric cardiologists do, postpones appropriate care and frequently discourages the adolescent, anxious to shed childhood and parental surveillance, from attending hospital. The paediatric cardiologist rarely has suitable inpatient facilities for these adult patients and many adolescents dislike remaining in children's wards.

The thought that "to understand frogs one should study tadpoles" is worth while. The cardiologist has the basic training and philosophy for coping with adult problems as well as with specialised cardiac care involving myocardial failure and arrhythmias. Unfortunately, the current generation of consultant cardiologists and juniors in training possess confidence but lack knowledge and experience and often interest in the complexities of congenital heart disease. A solution is to include some exposure to congenital heart disease, not necessarily in neonates or infants, in specialist cardiology training. Such patients provide excellent teaching material for the revival of the rapidly declining art of bedside cardiology. After all, exposure to adult cardiology is recommended in the training of a paediatric cardiologist, so why not the converse?

Just as newborn infants should be concentrated in special centres, so these adolescents and adults with congenital heart disease may best be helped by management in supraregional or a few regional centres with close links to the paediatric cardiac centres. These are cogent reasons for keeping the two specialities close to one another and not promoting the separate development that has occurred in North America. We are fortunate that in the United Kingdom our meetings, societies, and philosophy are interrelated.

The same principles apply to cardiac surgery, since many of these young patients with congenital heart disease require reoperation and too often their fate is determined by a good "adult" cardiac surgeon who does not know the disease or the traps into which he so easily may fall. There is a place for a few paediatric cardiac surgeons with the expertise and experience to deal with the problems of their patients as adults. This will increase continuity of care.

Referring doctors need to know that these are special patients, who usually require specialised knowledge that is not necessarily available in the local cardiac centre. Some supraregional centres should provide hospital beds for adolescents, and these should be made available to paediatric and adult cardiologists as well as to other specialities with similar patients who survive after chronic care during childhood. Overall care should be conducted by specially informed cardiologists, since individuals with congenital heart disease will spend most of their patient years as adults.

During the next decade fetal echocardiography and improved understanding of the causes of congenital heart diseases should reduce the numbers of children born with congenital anomalies. With improved and more courageous cardiac surgery, however, more adolescents and adults with congenital heart disease will survive.

I hope that an awareness of the problems may bring solutions. Today's problems will not be those of tomorrow, but while they are with us and until we can prevent congenital heart disease, we must provide expert lifetime care for the survivors. 